\title{
An Intertemporal Equilibrium Beta Pricing Model
}

\author{
Gregory Connor \\ University of California, Berkeley \\ Robert A. Korajczyk \\ Northwestern University
}

This article develops an intertemporal, discrete-time, competitive equilibrium version of the arbitrage pricing theory (APT) and explores the econometric implications of this model under various restrictions on investor preferences and on the dymamic bebavior of dividends. We describe conditions under wbicb the econometric techniques typically used for estimating and testing the APT can be sbown to be consistent with our economic model. We relate our intertemporal version of the APT to the static APT and to Merton's intertemporal capital asset pricing model.

We develop an intertemporal, discrete-time, competitive equilibrium version of Ross's arbitrage pricing theory (APT) and discuss the econometric content of this model under various restrictions on investor preferences and on the multivariate stochastic process determining dividends. We also discuss the distinctions between this model, the static APT, and the intertemporal capital asset pricing model (ICAPM) of Merton (1973).

The key distinction from the static APT is that corporate dividends, rather than equity returns, are assumed to obey an approximate factor model. The factor model on equity returns as well as the APT restriction on asset expected returns is derived endogenously.

The authors thank Gary Chamberlain, George Constantinides, Robert Hodrick, Ravi Jagannathan, Juan Ketterer, Daniel Siegel, an anonymous referee, Jonathan Ingersoll, and Michael Brennan for helpful comments. All remaining errors are our responsibility. Address reprint requests to Dr. Korajczyk, Kellogg Graduate School of Management, Northwestern University, 2001 Sheridan Road, Evanston, IL 60208-2006.

The Review of Financial Studies 1989 Volume 2, number 3, pp. 373-392

(C) 1990 The Review of Financial Studies 0893-9454/90/ $\$ 1.50$ 
The original arbitrage pricing theory of Ross (1976) is a static asset pricing theory. This means that one views the pricing equilibrium as occurring only once, followed by a terminal realization of investor wealth. Of course, the model is usually tested by relying on time-series data-that is, the observation of the repeated price setting process that occurs in security markets. The time-series data must be assumed to have various stationarity properties to render the model estimable. Since the asset pricing model is static (it has no time dimension), the appropriateness of these stationarity assumptions cannot be addressed within the pricing model. In this case, the economic model provides little guidance about the appropriate specification for the time-series properties of the statistical model.

Lucas (1978) suggests an integrated approach in which the statistical model used for estimation (a time-stationary relationship) is derived endogenously in a time-stationary, infinite-horizon asset pricing model. This approach was extended by Prescott and Mehra (1980), who named the approach a recursive competitive equilibrium (RCE) approach.

We apply the RCE approach to the APT, using a model that is similar to the RCE model that Bossaerts and Green (1988) developed in their intertemporal extension of the CAPM. We analyze whether the econometric assumptions needed to estimate the model can be derived endogenously within the model.

This general version of the intertemporal APT produces the APT pricing relationship at each point in time, but its time-series properties may not be consistent with the econometric assumptions generally used to estimate various versions of the model. Most, if not all, APT empirical tests require that asset returns obey a strict or approximate factor model with timeconstant betas [e.g., Roll and Ross (1980), Chen (1983), Connor and Korajczyk (1988), and Lehmann and Modest (1988)]. Many of the econometric procedures used in these empirical tests also require that returns be intertemporally homoskedastic. The general version of the intertemporal APT we present is not necessarily consistent with these econometric assumptions. Requiring consistency between the pricing model and the econometric assumptions makes the economic modeling problem more difficult and the necessary assumptions stricter.

We believe our RCE model is a useful complement to recent empirical studies that present evidence of time-varying risk premia [e.g., Keim and Stambaugh (1986); Fama and French (1988); French, Schwert, and Stambaugh (1987)]. The concept of time-varying risk premia cannot be properly explored within a static model such as the CAPM or static APT. Our competitive equilibrium approach has the potential to provide closed-form derivations of some of the econometric specifications for time-varying premia that these papers employ. In particular, we describe two special cases of our model that provide simple closed-form solutions and are consistent with estimation techniques that assume time-invariant parameters. One of the special cases predicts time-varying risk premia that behave in a manner 
similar to the premia found by Keim and Stambaugh (1986); Fama and French (1988); and French, Schwert, and Stambaugh (1987).

In Section 1 we prove the general version of the intertemporal equilibrium APT. Two special cases of the model that are consistent with timeinvariant parameters are developed in Section 2. In the first version, the representative investor has constant absolute-risk aversion while dividends are given by an approximate factor model and are independently and identically distributed through time. In the second, the representative investor has constant relative-risk aversion and the proportional changes (rather than levels) of dividends are independent and identically distributed through time. Section 3 describes the econometric distinctions among three beta pricing models: the static APT, the intertemporal APT, and Merton's ICAPM. Section 4 summarizes the article.

\section{An Intertemporal Competitive Equilibrium Version of the APT}

In this section we develop an intertemporal extension of the competitive equilibrium version of the APT presented in Connor (1984). The economy consists of an infinite number of identical investors. ${ }^{1}$ The investors live forever and have an additively separable, time-independent von NeumannMorgenstern utility function for the one good produced each period; the price of this good is normalized to 1 each period. Since all investors are identical, we act as if there were a single representative investor with timeseparable utility function $u(\cdot)$ and discount factor $\rho$.

There exists a countable infinity of corporations in the economy, all of which are purely equity financed. The per-share dividends of firm $i$ at time $t$ will be denoted $\tilde{x}_{i t}$, where $\sim$ denotes a random variable. The $R^{\infty}$-vector of corporate dividends at time $t$ follows an approximate factor model:

$$
\tilde{\mathbf{x}}_{t}=\mathbf{c} f_{0 t-1}+B \tilde{\mathbf{f}}_{t}+\tilde{\boldsymbol{\epsilon}}_{t}
$$

where $\mathbf{c}$ is an $R^{\infty}$-vector proportional to the vector of expected dividends (conditional on time $t-1$ information), $f_{0 t-1}$ is a scalar known at time $t$ $-1, B$ is an $R^{\infty} \times k$-matrix of asset betas, $\tilde{\mathbf{f}}_{t}$ is a $k$-vector stochastic process with $E_{t-1}\left(\tilde{\mathbf{f}}_{t}\right)=\mathbf{0}$, and $\tilde{\boldsymbol{\epsilon}}_{t}$ is an $R^{\infty}$-vector stochastic process with $E_{t-1}\left(\tilde{\boldsymbol{\epsilon}}_{t}\right)=$ 0. $E_{t-1}(\cdot)$ denotes expectations conditional on information at time $t-1$. In this section we impose weak assumptions on the stochastic processes $\tilde{\mathbf{f}}$ and $\tilde{\boldsymbol{\epsilon}}$.

In the simplest version of the model, $f_{0 t-1}=1$ for all $t$ so that the expected dividends to the equities are constant through time. However, we also allow $f_{0 t-1}$ to be a time trend (deterministic growth) or a stochastic process (random growth in expected dividends). Note that $f_{0 t-1}$ is known at time $t-1$, and so we can speak of $c_{0 t-1}$ as the expected dividends for time $t$ given the information available at time $t-1$.

${ }_{1}^{1}$ Conditions for aggregation are considered in Bossaerts and Green (1987). 
A portfolio $\boldsymbol{\alpha}_{t-1}$ is a linear combination of the assets such that the portfolio payoff $\boldsymbol{\alpha}_{t-1}^{\prime} \tilde{\mathbf{x}}_{t}$ has finite second moment. We permit the investor to hold "limit" portfolios, which are infinite dimensional linear combinations of the countable set of assets, as in Connor $\left(1984\right.$, p. 16). ${ }^{2}$ The portfolio for period $t$ is determined at the beginning of the period and, therefore, is dated at time $t-1$.

The market portfolio $\mathbf{q}$ is the per-capita supply of the assets. Since no new shares are issued or redeemed in the economy, $\mathbf{q}$ is constant through time. Let $\mathbf{p}_{t}$ denote the $R^{\infty}$-vector of asset prices at time $t$, with the price of the $i$ th firm given by $p_{i r}$.

We utilize the following set of assumptions:

Assumption 1. There exists an infinite-lived representative investor with a risk-averse von Neumann-Morgenstern utility function $u(\cdot)$ and time discount factor $\rho$. That is, the investor wishes to maximize expected lifetime utility

$$
E_{t}\left[\sum_{s=1}^{\infty} \rho^{s} u\left(\tilde{C}_{t+s}\right)\right]
$$

subject to the budget constraint

$$
\boldsymbol{\alpha}_{t-1}^{\prime} \mathbf{x}_{t}+\boldsymbol{\alpha}_{t-1}^{\prime} \mathbf{p}_{t} \geq \boldsymbol{\alpha}_{t-1}^{\prime} \mathbf{p}_{t}+\mathbf{C}_{t}
$$

Assumption 2. There exists a countable infinity of purely equity-financed firms with net per-share dividends given by Equation (1).

\section{Assumption 3.}

$$
E_{t-1}\left[\tilde{\boldsymbol{\epsilon}}_{t} \mid \mathbf{f}_{t}\right]=\mathbf{0}
$$

Let $B^{* n}$ denote the $n \times(k+1)$ matrix $\left[B^{n} \mid c^{n}\right]$ where $B^{n}$ and $c^{n}$ denote the first $n$ rows of $B$ and $c$. Also, let $\tilde{\epsilon}_{t}^{n}$ denote the first $n$ rows of $\tilde{\epsilon}_{t}$ and \|\| denote the matrix $L_{2}$-norm.

\section{Assumption 4.}

$$
\lim _{n \rightarrow \infty}\left\|\left(B^{* n^{\prime}} B^{* n}\right)^{-1}\right\|<\infty
$$

Assumption 5.

$$
\lim _{n \rightarrow \infty}\left\|E_{t-1}\left[\tilde{\epsilon}_{t}^{n} \tilde{\epsilon}_{t}^{n \prime}\right]\right\|<\infty
$$

Assumption 6.

$$
E_{t-1}\left[\left(\mathbf{q}^{\prime} \tilde{\boldsymbol{\epsilon}}_{t}\right)^{2}\right]=0
$$

2 The space of portfolios is the Hilbert space of linear combinations of $x_{t p} i=1,2, \ldots$ under the meansquare norm $\left\|\alpha_{t-1}\right\|=E_{t-1}\left[\left(\alpha_{t-1}^{\prime} x_{t}\right)^{2}\right]$. 


\section{Assumption 7.}

$$
\lim _{s \rightarrow \infty} E_{t-1}\left[\rho^{s} u^{\prime}\left(\mathbf{q}^{\prime} \mathbf{x}_{t+s}\right)\left(\tilde{x}_{i t+s}+\tilde{p}_{i t+s}\right)\right]=0 \quad \text { for all } i=1,2, \ldots
$$

Assumptions 1 and 2 were described above. Note that Assumption 2 applies to dividends paid out rather than total profits. Reinvestment of profits is permitted in the model as long as the cash paid out follows the assumed factor model. We do not explicitly model the reinvestment decision and so treat dividends as exogenous.

Assumption 3 states that the idiosyncratic risks are risky in the sense of Rothschild and Stiglitz (1970). This guarantees that a risk-averse investor holding a well-diversified portfolio will not want to hold any $\epsilon$ risk [see Connor (1984) for more discussion on this point]. Assumptions 4 and 5 are the fundamental assumptions of an approximate factor model. Assumption 4 guarantees that the factor risks are pervasive in the economy; Assumption 5 guarantees that the idiosyncratic risks can be eliminated from a portfolio by spreading its weights "evenly" across many assets [e.g., see Chamberlain and Rothschild (1983) and Ingersoll (1984)]. Intuitively, Assumption 4 means that the factor risks are the economywide sources of risk and Assumption 5 means that the idiosyncratic risks are the firm- or industry-specific sources of risk.

Assumption 6 requires that the market portfolio be well-diversified. This is a key assumption behind the competitive equilibrium version of the APT. Assumption 7 is a transversality condition that guarantees that no asset has infinite value to the representative investor.

The following is an intertemporal extension of Connor (1984, theorem 3).

Tbeorem 1. Given Assumptions $1-7$, there exists a unique sequence of competitive equilibrium prices given by

$$
\begin{aligned}
\mathbf{p}_{t}= & \mathbf{c} \pi_{0 t}+B \pi_{t} \\
\pi_{0 t}= & \frac{E_{t}\left[\sum_{s=1}^{\infty} \rho^{s} \mathcal{u}^{\prime}\left(\mathbf{q}^{\prime} \tilde{\mathbf{x}}_{t+s}\right) f_{0 t+s-1}\right]}{u^{\prime}\left(\mathbf{q}^{\prime} \mathbf{x}_{t}\right)} \\
\pi_{j t}= & \frac{E_{t}\left[\sum_{s=1}^{\infty} \rho^{s} u^{\prime}\left(\mathbf{q}^{\prime} \tilde{\mathbf{x}}_{t+s}\right) \tilde{f}_{j t+s}\right]}{u^{\prime}\left(\mathbf{q}^{\prime} \mathbf{x}_{t}\right)}
\end{aligned}
$$

where $\pi_{o t}$ is a scalar and $\pi_{t}$ is a $k \times 1$ vector whose jth element is $\pi_{j t}$.

Proof. See the Appendix.

We have proved that prices are beta linear: The price of each asset is a linear multiple of its expected dividend and its sensitivities to the $k$ factors, 
$\mathbf{B}_{i} .{ }^{3}$ This is the basic prediction of the APT. Note, however, that the "factor prices" $\pi_{0 t}$ and $\pi_{i}$ need not be constant through time.

In order to render Equation (2) compatible with time-series return data, we must rewrite the relation in terms of rates of return. Define the $R^{\infty}$. vector $\mathbf{r}_{t}$ whose $i$ th element is given by $\left(p_{i t}+x_{i t}\right) / p_{i t-1}$. In order to perform this division we must be sure that $p_{i t} \neq 0$ for all $i$ and $t$, but this is not a substantive problem. ${ }^{4}$

Let $\boldsymbol{\alpha}_{m 1}$ denote a well-diversified portfolio with $\boldsymbol{\alpha}_{m 1}^{\prime} \mathbf{c}=0$ and $\boldsymbol{\alpha}_{m 1}^{\prime} B=(1$, $0, \ldots, 0)$. We will call this portfolio the first factor portfolio since it mimics the first factor. Similarly, define $\boldsymbol{\alpha}_{m 2}, \ldots, \boldsymbol{\alpha}_{m k}$ to mimic the factors $2, \ldots$, $k$. Note that $\boldsymbol{\alpha}_{m j}^{\prime} \mathbf{p}_{t}=\pi_{j t}$ and $\boldsymbol{\alpha}_{m j}^{\prime} \mathbf{x}_{t}=f_{j t}$. Define $r_{m 1 t}, \ldots, r_{m k t}$ as the gross returns to these portfolios $\left[r_{m j t}=\left(\pi_{j t}+f_{j t}\right) / \pi_{j t-1}\right]$. Denote the zero-beta portfolio as a well-diversified portfolio such that $\boldsymbol{\alpha}_{0}^{\prime} \mathbf{c}=1, \boldsymbol{\alpha}_{0}^{\prime} B=(0, \ldots$, 0 ), and the return on this portfolio, $r_{0 t}$, as the zero-beta return. Connor (1984, lemma 3) shows that given Assumptions 2-5, these portfolios exist. The beta pricing result of Theorem 1 can be restated in rate-of-return form using these portfolios.

Corollary 1. Given Assumptions 1-7 and the additional assumption that $f_{o t+s}$ is independent of $\tilde{\epsilon}_{t}$ for all $s \geq 0$, then

$$
\begin{array}{rl}
\tilde{\mathbf{r}}_{t} & =\iota \tilde{r}_{0 t}+\boldsymbol{\beta}_{1 t-1}\left(\tilde{r}_{m 1 t}-\tilde{r}_{0 t}\right)+\cdots+\boldsymbol{\beta}_{k t-1}\left(\tilde{r}_{m k t}-\tilde{r}_{0 t}\right)+\tilde{\epsilon}_{i}^{*} \\
\boldsymbol{\beta}_{j t}^{\prime} & =\left(\beta_{1 j t}, \beta_{2 j t}, \ldots\right) \\
\iota^{\prime} & =(1,1,1, \ldots) \\
\beta_{i j t} & =\frac{B_{i j} \pi_{j t}}{c_{i} \pi_{0 t}+B_{i 1} \pi_{1 t}+\cdots+B_{i k} \pi_{k t}} \quad j=1, \ldots, k, i=1,2, \ldots \\
\tilde{\epsilon}_{t t}^{*} & =\frac{\tilde{\epsilon}_{i t}}{c_{i} \pi_{0 t-1}+B_{i 1} \pi_{1 t-1}+\cdots+B_{i k} \pi_{k t-1}} \quad i=1,2, \ldots \\
E\left(\tilde{\epsilon}_{i t}^{*} \mid r_{0 t}, r_{m 1 t}, \ldots, r_{m k t}\right)=0 & i=1,2, \ldots
\end{array}
$$

Proof. See the Appendix.

Corollary 1 assumes that the factor risk prices $\pi_{j t}$ are nonzero for all $j$ and $t$. If $\pi_{j t}=0$ for some $j$ and $t$, a minor adjustment must be made to the definitions of $r_{m j t}$ and $\boldsymbol{\beta}_{j t .}{ }^{5}$ Equation (3) can be interpreted as a regression

\footnotetext{
${ }_{3}^{3} \mathbf{B}_{i}$ denotes row $i$ of the matrix $B$, while $\mathbf{B}_{f}$ denotes column $j$.

${ }^{4}$ The simplest solution is to assume that $x_{i t}>0$ for all $i$ and $t$, which is not inconsistent with our other assumptions. Alternatively, for assets with $p_{i t-1}=0$, we can redefine the assets' payoffs to be their current payoffs plus the payoff to a unit investment in the zero-beta asset (defined below). The return on the redefined asset is $\left(p_{i t}+x_{i t}+r_{0 t}\right) / 1$.

${ }^{5}$ If $\pi_{y t}=0$, then define $\beta_{t j t}=B_{i j} /\left(c_{i} \pi_{0 t}+B_{i \times 1 t}+\cdots+B_{i k} \pi_{k t}\right), i=1,2, \ldots$ and $r_{m j t}=\pi_{j t}-\pi_{j t-1}+f_{j t}+r_{0 t}$ Equation (3) remains unchanged.
} 
model where the explanatory variables are the excess returns on the $k$ reference portfolios (excess relative to the zero-beta return).

In our multiperiod model, the zero-beta return differs from the oneperiod riskless return. Let $r_{F t-1}$ denote the gross return on the one-period riskless asset, that is, the return from $t-1$ to $t$ on a security with a single certain payoff of 1 at $t .^{6}$ We assume that the riskless asset is in zero net supply. By the same logic used to derive Equation (2), the riskless asset's return is given by

$$
r_{F t-1}=\frac{u^{\prime}\left(\mathbf{q}^{\prime} \mathbf{x}_{t-1}\right)}{\rho E_{t-1}\left[u^{\prime}\left(\mathbf{q}^{\prime} \tilde{\mathbf{x}}_{t}\right)\right]}
$$

In comparison, note that Equation (2) implies that the price, at $t-1$, of the zero-beta asset is $\pi_{0 t-1}$. This, in turn, implies that the zero-beta return is given by

$$
r_{0 t}=\frac{f_{0 t-1}}{\pi_{0 t-1}}+\frac{\tilde{\pi}_{0 t}}{\pi_{0 t-1}}
$$

Next period's dividend yield on the zero-beta asset is certain, but the capital gain on this asset is random. In a sense, $r_{0 t}$ represents a term structure factor. If $f_{0 t}$ evolves deterministically, then $r_{0 t}$ is equal to the return on a portfolio of discount bonds with payoffs equal to $f_{0 t+s-1}$ at time $t+s, s>$ 0 . In the next section we compare $r_{0 t}$ and $r_{F t-1}$ for two specific cases.

Note that Corollary 1 implies that the $k+1$ portfolios $r_{0 t}, r_{m 1 t}, \ldots, r_{m k t}$ span the mean-variance frontier of the assets described in Equation (1), since a regression of $r_{i t}$ on the $k+1$ portfolios has an intercept of zero and $k+1$ betas that sum to unity [see Huberman and Kandel (1987)]. Equivalently, Equation (3) implies that any asset's return can be constructed from a linear combination of these $k+1$ portfolios plus a zeromean idiosyncratic term $\tilde{\boldsymbol{\epsilon}}^{*}$.

Ohlson and Garman (1980) also investigate the properties of an intertemporal APT. They assume that factor risk premia and the riskless return are constant through time. In our model the premia and riskless rate vary through time, in general. We give sufficient conditions for constant premia and riskless return below.

\section{Two Special Cases}

The two pricing models presented in this section are special cases of the model in Theorem 1. In each, the process determining dividends and the preferences of the representative investor are restricted. Both models have asset betas that are constant through time, and in the second model idiosyncratic returns are homoskedastic.

${ }_{6}^{6}$ The one-period riskless return from $t-1$ to $t$ is dated $t-1$ since the return is observed at $t-1$ 
We are interested in conditions for constant betas and homoskedasticity since these conditions are commonly assumed in applications of the APT. For example, restrictions implied by the asset pricing models on the parameters of time-series regressions of asset returns on factor-mimicking portfolio returns are used to test these models. It is commonly assumed in empirical work that the betas of these regressions are constant. Conditional on having the factor-mimicking portfolios, it may be possible to incorporate time variation of the betas directly into the estimation procedure [e.g., Bollerslev, Engle, and Wooldridge (1988)]. A more difficult issue is the fact that many procedures used to construct factor-mimicking portfolios (such as factor analysis or principal components) implicitly assume a timeinvariant return-generating process. Since much of the empirical literature relies on this assumption, it seems important to investigate the types of economies that might be consistent with constant betas and/or homoskedasticity.

In the first model we assume that the representative investor has constant absolute-risk aversion ${ }^{7}$ and dividends are independently and identically distributed through time.

Assumption 8. The representative investor's utility function is $u\left(C_{\downarrow}\right)=$ $-(1 / \gamma) e^{-\gamma c_{t}}$

Assumption 9. The stochastic process $\tilde{\mathbf{f}}_{t}$ is independently and identically distributed through time and $f_{o t-1}=1$ for all $t$.

Corollary 2. Given the assumptions of Corollary 1 plus Assumptions 8 and 9, there exists a competitive equilibrium such that Equation (3) bolds with $\boldsymbol{\beta}_{j}$ constant through time for $j=1,2, \ldots, k$.

Proof. See the Appendix.

Note that the risk premia associated with the factor-mimicking portfolios are time-varying. That is,

$$
\begin{aligned}
\beta_{i j} E_{t-1}\left(\tilde{r}_{m j t}-\tilde{r}_{0 t}\right)= & \phi_{i j} u^{\prime}\left(\mathbf{q}^{\prime} \mathbf{x}_{t-1}\right) \\
\phi_{i j}= & \left(\frac{B_{i j} \mu_{j}}{\mu_{0}}\right)\left(\frac{\rho-1}{\rho}\right) \\
& \cdot\left(\frac{1}{c_{i} \mu_{0}+B_{i 1} \mu_{1}+\cdots+B_{i k} \mu_{k}}\right)
\end{aligned}
$$

where $\mu_{0}=E_{t-1}\left[u^{\prime}\left(\mathbf{q}^{\prime} \tilde{\mathbf{x}}_{t}\right)\right]$ and $\mu_{j}=E_{t-1}\left[u^{\prime}\left(\mathbf{q}^{\prime} \tilde{\mathbf{x}}_{t}\right) \tilde{f}_{j t}\right]$. Note that, by our i.i.d. (independently and identically distributed) assumption, $\mu_{0}, \mu_{j}(j=1,2$, $\ldots, k)$, and, therefore, $\phi_{i j}$ are independent of time. Without loss of gen-

\footnotetext{
'Actually, the corollary holds for general utility functions. This can be seen by inserting $u^{\prime}\left(C_{t}\right)$ for exp $\left(-\gamma C_{t}\right)$ in the proof of Corollary 2. This is shown in Bossaerts and Green (1987) and was also suggested to us by Ravi Jagannathan.
} 
erality the factors can be defined such that $\mathbf{q}^{\prime} \mathrm{B}>0$. This implies that $\boldsymbol{\mu}_{j}$ $<0$ (because of decreasing marginal utility) and that the sign of $\phi_{i j}$ is equal to the sign of $B_{i j}$. The result is intuitive. When current output $\left(\mathbf{q}^{\prime} \mathbf{x}_{t-1}\right)$ is high, marginal utility is low, and hence the representative agent requires less compensation for bearing positive factor $j$ type risk. This result is consistent with some of the empirical literature on time variation in risk premia. Note that $\mathbf{p}_{t-1}$ is a function of $\mathbf{q}^{\prime} \mathbf{x}_{t-1}$ through $\pi_{0 t-1}$ and $\boldsymbol{\pi}_{t-1}$. High current output leads to high prices of the assets. Thus, the model predicts an inverse relation between risk premia and current prices. Keim and Stambaugh (1986) find such an inverse relation in bond and stock returns. There is a positive relation between risk premia and dividend yields. This is consistent with the empirical findings of Fama and French (1988). The model also leads to some predictions that are not consistent with empirical evidence. There is strong mean reversion in asset prices, since $E_{t}\left[\tilde{\mathbf{p}}_{t+s}\right]$ (for $s>0$ ) does not depend on $\mathbf{p}_{t}$ (i.e., prices are i.i.d.). While there is evidence of some mean reversion in stock prices [see Lo and MacKinlay (1988)], it is a second-order effect. Also, the time-varying risk premia are proportional to the gross return on a one-period riskless asset, $r_{F t-1}$. In Section 1 we showed that $r_{F t-1}=u^{\prime}\left(\mathbf{q}^{\prime} \mathbf{x}_{t-1}\right) / \rho E_{t-1}\left[u^{\prime}\left(\mathbf{q}^{\prime} \tilde{\mathbf{x}}_{t}\right)\right]$, which is proportional to $u^{\prime}\left(\mathbf{q}^{\prime} \mathbf{x}_{t-1}\right)$ under our i.i.d. assumption $\left\{\right.$ since $E_{t-1}\left[u^{\prime}\left(\mathbf{q}^{\prime} \tilde{\mathbf{x}}_{t}\right)\right]$ is independent of $t$ in that case $\}$. This prediction is inconsistent with the evidence in Fama and Schwert (1977), which indicates an inverse relation between the risk premium on common stock and short-term Treasury bills.

Thus, this model is one with constant betas and time variation in risk premia. An additional implication of the model is that the idiosyncratic variability of asset returns depends on $\left[\boldsymbol{u}^{\prime}\left(\mathbf{q}^{\prime} \mathbf{x}_{t-1}\right)\right]^{2}$. That is, if

$$
E_{t-1}\left[\tilde{\epsilon}_{t} \tilde{\epsilon}_{t}^{\prime} \mid \mathbf{f}_{t}\right]=V_{t}
$$

then

$$
E_{t-1}\left[\tilde{\epsilon}_{t} \tilde{\epsilon}_{t}^{\prime} \mid \tilde{\mathbf{f}}_{t}\right] \propto\left[u^{\prime}\left(\mathbf{q}^{\prime} \mathbf{x}_{t-1}\right)\right]^{2} V_{t}
$$

Thus, even if the the errors in Equation (1) are temporally homoskedastic $\left(V_{t}=V\right.$, for all $t$ ), the errors in Equation (3) are conditionally heteroskedastic. Note that the model predicts a positive relationship between timevarying risk premia and time-varying idiosyncratic variance. This is consistent with the empirical findings of French, Schwert, and Stambaugh (1987).

Corollary 2 provides an econometrically estimable version of the APT, but it uses the unattractive assumption that corporate dividends are intertemporally independent in their levels. An increase in corporate dividends this period has no effect on expected future dividends. A more attractive assumption is intertemporal independence in the rates of change of corporate dividends. The next model leads to i.i.d. rates of change in corporate dividends and conditional homoskedasticity of idiosyncratic returns.

We let the dividends to firms follow an i.i.d. approximate factor model scaled by last period's per-capita dividends. That is, 


$$
\tilde{\mathbf{x}}_{t}=\left(\mathbf{c}+B \tilde{\mathbf{f}}_{t}+\tilde{\boldsymbol{\epsilon}}_{t}\right)\left(\mathbf{q}^{\prime} \mathbf{x}_{t-1}\right)
$$

For convenience we assume that $\mathbf{q}^{\prime} \mathbf{c}=1$, so that the average growth rate in per-capita consumption is zero. We assume that $\mathbf{q}^{\prime} B \tilde{\mathbf{f}}$, is bounded above -1 , so that per-capita dividends are never negative [although Equation (4) does not necessarily preclude negative dividends on individual firms]. Note that per-capita dividends are intertemporally independent in proportional changes. This assumed nonstationarity in dividends is consistent with the evidence reported in Kleidon (1986). We also assume that the representative investor has constant relative-risk aversion.

Assumption 10. Corporate dividends are given by Equation (4).

Assumption 11. The utility function of the representative investor is $u\left(C_{\nu}\right)$ $=\left(C_{t}^{1-\gamma}-1\right) /(1-\gamma)$ with $\gamma \geq 0$.

Assumption 12. $E_{t-1}\left[\tilde{\epsilon}_{\hat{t}} \tilde{t}_{t}^{\prime} \mid \mathbf{f}_{t}\right]=V$, where $V$ is independent of $t$.

Corollary 3. Given the assumptions of Corollary 1, with Assumption 2 replaced by Assumption 10, plus Assumptions 11 and 12, there exists a unique sequence of competitive equilibrium returns given by Equation (3) where $\boldsymbol{\beta}_{j}$ is constant througb time for $j=1,2, \ldots, k$, and $\tilde{\boldsymbol{\epsilon}}_{t}^{*}$ is homoskedastic.

Proof. See the Appendix.

In this version of the model the factor risk premia and the one-period riskless return $r_{F t-1}$ do not vary through time, so there is no relation between the risk premia and $r_{F t-1}$. To see that $r_{F t-1}$ is constant note that

$$
\begin{aligned}
r_{F t-1} & =\frac{u^{\prime}\left(\mathbf{q}^{\prime} \mathbf{x}_{t-1}\right)}{\rho E_{t-1}\left[u^{\prime}\left(\mathbf{q}^{\prime} \tilde{\mathbf{x}}_{t}\right)\right]} \\
& =\frac{\left(\mathbf{q}^{\prime} \mathbf{x}_{t-1}\right)^{-\gamma}}{\rho\left(\mathbf{q}^{\prime} \mathbf{x}_{t-1}\right)^{-\gamma} E_{t-1}\left[1+\mathbf{q}^{\prime} B \tilde{\mathbf{f}}_{t}\right]^{-\gamma}} \\
& =\frac{1}{\rho E_{t-1}\left[1+\mathbf{q}^{\prime} B \tilde{\mathbf{f}}_{t}\right]^{-\gamma}}
\end{aligned}
$$

Thus, given our i.i.d. assumption on $\mathbf{f}_{t}$, the expectation in the denominator is constant through time. Similar analysis shows that the risk premia are constant through time. Also, $E_{t-1}\left[\tilde{r}_{0 t}\right]=r_{F t-1}$.

We have now produced two particular models with time-constant betas; both involve strong assumptions. It seems appropriate to ask how much more general a model could be and still preserve this property. A key expression is $r_{i t}=\left(p_{i t}+x_{i t}\right) / p_{i t-1}$, defining the time $t$ return of asset $i$. The time $t-1$ price of the asset appears in the denominator, whereas the 
numerator includes both the time $t$ dividend of the asset and the time $t$ price change. In any recursive, competitive equilibrium model, both the numerator and denominator of this expression will be random through time. Given an exogenously assumed structure of $\mathbf{x}_{t}$ and endogenously determined structure of $\mathbf{p}_{t}$ we must define a factor model for these returns [such as Equation (3)] in which the betas of the assets are constant. In order for the betas to be constant, the randomness in the numerator and denominator of the rate-of-return expression must be offsetting. It is this strict requirement that gives rise to our strong assumptions.

\section{A Comparison of Three Beta Pricing Models}

In this section we describe the testable content of the intertemporal APT developed above and compare it to the static APT and the ICAPM. ${ }^{8}$ We argue that, in principle, these three models are distinct, and we describe the distinctions between them. We conjecture that in practice it may be difficult to distinguish them empirically.

The static equilibrium APT and the assumption of constant betas imply that asset returns have the form

$$
\tilde{r}_{i t}=r_{F t-1}+\boldsymbol{\beta}_{i} \cdot\left(\tilde{\mathbf{r}}_{m t}-\iota r_{F t-1}\right)+\tilde{\boldsymbol{\epsilon}}_{i t}
$$

where $\boldsymbol{\beta}_{i .}=\left(\beta_{i 1}, \ldots, \beta_{i k}\right)$ and $\mathbf{r}_{m t}=\left(r_{m 11}, \ldots, r_{m k t}\right)^{\prime}$. Subtracting $r_{F t-1}$ from both sides of (5) to give an equation for asset excess returns and also expressing the mimicking portfolio returns as excess returns gives

$$
\tilde{R}_{i t}=\boldsymbol{\beta}_{i} \cdot \tilde{\mathbf{R}}_{m t}+\tilde{\epsilon}_{i t}
$$

where $R_{i t}=r_{i t}-r_{F t-1}$ and $\mathbf{R}_{m t}=\mathbf{r}_{m t}-\iota r_{F t-1}$. This key equation of the static APT is testable by a variety of procedures [see, for example, Connor and Korajczyk (1988) and Lehmann and Modest (1988)].

There are at least three differences between the static [Equation (5)] and intertemporal [Equation (3)] versions of the APT. First, the beta coefficients of the intertemporal version have a time subscript. While the static model does not preclude time-varying parameters, it also provides no guidance about the nature of the time variation. In statistical implementations, where the model must be estimated with time-series data, most analysts implicitly assume that betas are constant. This implicit assumption may not hold, however, when the time dimension is included explicitly as we have done. A second difference between the intertemporal and static versions is that the intertemporal risk premia may vary through time. Again, since the static model does not consider the time dimension, it makes no prediction about the nature of the time variation in risk premia.

The third distinction from the static model is the long-run nature of the zero-beta asset return $\tilde{r}_{0 t}$ relative to the one-period riskless return $r_{F t-1}$. This induces a restriction on the matrix of factor sensitivities when excess returns

${ }^{8} \overline{\text { Constantinides (1989) provides a discussion of relations between the versions of the APT and the ICAPM. }}$ 
are calculated relative to the one-period riskless return. To see this, use $R_{i t}=r_{i t}-r_{F t-1}$ and Equation (3) to express returns in excess of the oneperiod risk-free return as

$$
\tilde{R}_{i t}=\left(\tilde{r}_{0 t}-r_{F t-1}\right)+\beta_{i, t-1}\left(\tilde{\mathbf{r}}_{m t}-\imath \tilde{r}_{0 t}\right)+\tilde{\epsilon}_{i t} \quad i=1,2, \ldots
$$

Let $\boldsymbol{\beta}_{i \cdot t-1}^{*}=\left(1, \boldsymbol{\beta}_{i \cdot t-1}\right)$ and $\mathbf{R}_{m t}^{* \prime}=\left(\left(r_{0 t}-r_{F t-1}\right), \mathbf{R}_{m t}^{\prime}\right)$. We can write Equation (7) as

$$
\tilde{R}_{i t}=\boldsymbol{\beta}_{i \cdot t-1}^{*} \tilde{\mathbf{R}}_{m t}^{*}+\tilde{\epsilon}_{i t}
$$

which is similar to the estimable Equation (6) except that the number of factors has increased from $k$ to $k+1$ and the first column of $\beta_{t-1}^{*}$ is equal to the unit vector $\iota$ (and we have allowed for time variation in $\beta_{t-1}^{*}$ ). In this way, we add a "term structure" factor to the model and produce an equation similar to the static APT."

We can test for the difference between the static and intertemporal versions of the APT by testing the unit-beta restriction of the intertemporal model. ${ }^{10}$ If we use multivariate statistical methods (such as factor analysis or principal components) to estimate $\mathbf{R}_{m t}^{*}$ then we cannot identify the particular column of $\beta_{t}^{*}$ that has the unit-beta constraint, since the model is subject to a rotational indeterminacy. Let L denote a $(k+1) \times(k+1)$ nonsingular matrix. Factor analytic methods cannot distinguish between the model $\beta_{t-1} \mathbf{R}_{t}^{*}$ and the model $\left(\beta_{t-1} \mathrm{~L}\right)\left(\mathrm{L}^{-1} \mathbf{R}_{t}^{*}\right)$. Allowing for this indeterminacy, the restriction placed upon $\beta^{*}$ is that there exists a $(k+1)$. vector $\boldsymbol{\lambda}$ such that

$$
\beta^{*} \lambda=\iota
$$

Assuming that the betas are time-invariant (as in Corollaries 2 and 3 ), this hypothesis can be tested as a nonlinear restriction on the coefficients of a multivariate regression model. The restriction is nonlinear because $\lambda$ is not known. Partition $\beta^{*}$ into $\beta^{* 1}$, a $(k+1) \times(k+1)$ matrix formed from the first $k+1$ rows of $\beta^{*}$, and $\beta^{* 2}$, a $(n-k-1) \times(k+1)$ matrix formed from the remaining rows. Then Equation (9) implies that $\beta^{* 2}\left(\beta^{* 1}\right)^{-1} \iota_{k+1}-$ $\iota_{n-k-1}=0 .{ }^{11}$ If the intertemporal zero-beta return is not observable, the static and constant-beta versions of the intertemporal APT are only distinct to the extent that the constraint of Equation (9) is violated [see Connor and Korajczyk (1988) for an implementation of this test].

Merton's ICAPM provides an alternative to either version of the APT. We will rely on a simple discrete-time analog of Merton's continuous-time model discussed more fully in Constantinides (1989). Let $J_{t}$ denote the

\footnotetext{
9 Jagannathan and Viswanathan (1988) also analyze term structure effects in linear factor models.

${ }^{10}$ It is possible in the static APT that the beta matrix could obey the unit-beta restriction. The difference is that the intertemporal model derives this restriction endogenously. In the static model one would need to exogenously assume that there exists a factor for which every asset has equal sensitivity.

1 The subscript on the unit vector $\iota$ denotes the length of the vector.
} 
derived utility of the representative investor at time $t$, and let $J_{W t}$ denote his marginal derived utility given a change in time $t$ wealth:

$$
J_{t}=E_{t}\left[\sum_{s=0}^{\infty} \rho^{s} u\left(\tilde{C}_{t+s}\right)\right] \quad J_{W t}=\frac{\partial J_{t}}{\partial W_{t}}
$$

Assume that we can express $J_{w t}$ as a linear combination of $k$ uncorrelated state variables: $J_{w_{t}}=z_{1 t}+\cdots+z_{k t}$. From a first-order condition of com petitive equilibrium at time $t-1$ we have

$$
u^{\prime}\left(C_{t-1}\right)=E_{t-1}\left[J_{w t} r_{i t}\right]
$$

for every asset return $r_{i t}$. Let $r_{m j t}(j=1, \ldots, k)$ denote a set of portfolio returns such that $r_{m j t}$ is perfectly negatively correlated with $z_{j t}$. Then it is easy to show ${ }^{12}$ that we can write Equation (10) as

$$
\tilde{r}_{i t}-\tilde{r}_{F t-1}=\beta_{i 1 t-1}\left(\tilde{r}_{m 1 t}-\tilde{r}_{F t-1}\right)+\cdots+\beta_{i k t-1}\left(\tilde{r}_{m k t}-\tilde{r}_{F t-1}\right)+\tilde{\epsilon}_{i t}
$$

where $\beta_{i j t-1}=\operatorname{cov}_{t-1}\left(\tilde{r}_{i t}, \tilde{r}_{m j t}\right) / \operatorname{var}_{i-1}\left(\tilde{r}_{m j t}\right)$ and $E\left[\tilde{\epsilon}_{i t}\right]=0$.

Our intertemporal APT can be viewed as a special case of this model. It has $k+1$ state variables: the realizations of the $k$ factors and the realization of the aggregate dividend. These state variables are spanned by the $k$ factormimicking portfolios and the zero-beta portfolio. [See Constantinides (1989) for a more general treatment of the ICAPM that allows for time-dependence in utility and an arbitrary number of state variables.]

Note the similarity between Equation (11) and the APT Equation (3). The basic difference between the models lies in the definition of the portfolio returns on the right-hand side of the equations for asset returns. In the APT, these portfolios mimic the pervasive factors in the covariance matrix of asset returns. The portfolio returns may be identified by factoranalytic methods applied to the cross section of asset returns [see, for example, Roll and Ross (1980), Chen (1983), Connor and Korajczyk (1988), or Lehmann and Modest (1988)]. In the ICAPM, the portfolio returns mimic the state variables that index the representative investor's derived utility of wealth function. The econometrician must go outside the basic model to identify the set of state variables and then construct mimicking portfolios for these state variables.

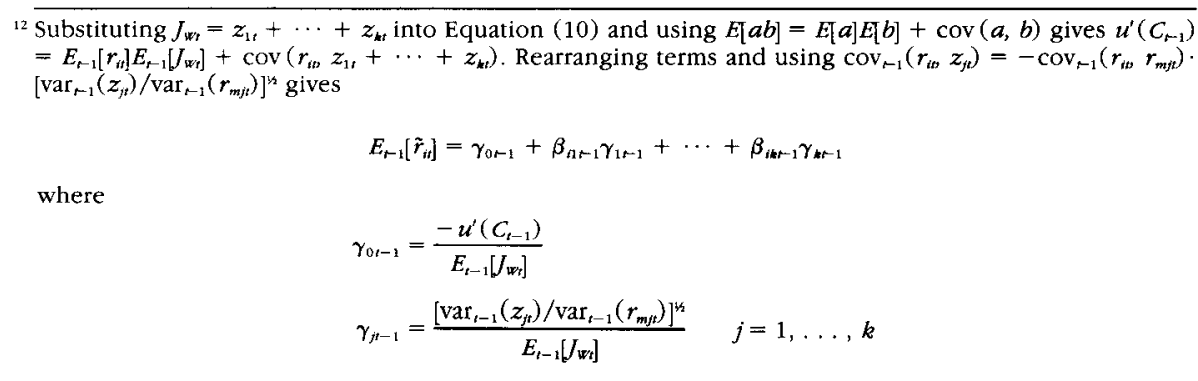

By substitution we get $\gamma_{0 r-1}=r_{F r-1}$ and $\gamma_{f r-1}=E_{t-1}\left[r_{m f t}-r_{F r-1}\right]$. Using these expressions and taking expec tations of Equation (11) gives the needed result that $E_{t-1}\left[\epsilon_{t i}\right]=0$. 
Let us summarize the empirical distinction between the APT and the ICAPM. The beta pricing restrictions [ Equations (11) and (7)] are identical in these two models. The only econometric distinction between the two models concerns the method the econometrician uses to identify the appropriate mimicking portfolios in Equations (7) or (11). For the APT, these mimicking portfolios are identified from the approximate factor model Assumptions 4 and 5 using factor-analytic techniques. The mimicking portfolios mimic the pervasive factors in asset returns. The ICAPM implies a different method. The mimicking portfolios are identified by theoretical criteria (not fully specified in the general form of the model). The mimicking portfolio must mimic a set of state variables that index the stochastic changes in the representative investor's wealth function. In terms of the final econometric specification, the difference in the identification of the mimicking portfolios is the only difference between the two models.

The ICAPM and APT are distinguishable in principle, but perhaps not in practice. There is certain to be an overlap, if not an identity, between the mimicking portfolios found by factor-analytic decomposition of asset returns and those found by exogenous choice of state variables. ${ }^{13}$ It may be that the set of state-variable portfolio returns of the ICAPM will be approximately equal to the factor-mimicking portfolios of the APT-in which case the APT and ICAPM are empirically indistinguishable. The static and intertemporal versions of the APT are distinguished by the unitbeta restriction of the intertemporal model and the time variation of some parameters in the intertemporal model.

\section{Summary}

This article applies the recursive, competitive equilibrium approach of Lucas (1978) and Prescott and Mehra (1980) to the APT. This approach requires the development of a competitive equilibrium asset pricing model that is consistent with the time-series statistical techniques used for estimation. A drawback to this approach is that this type of model usually requires strong assumptions. An advantage of the approach is that these assumptions are described explicitly, rather than being implicit in the empirical implementations of more general theoretical models.

The model incorporates one of the time-series return patterns uncovered in recent empirical asset pricing studies: the presence of time-varying risk premia. Although we do not fully explain all the empirically observed time-series patterns in risk premia, we believe that our approach is a useful first step toward explaining them within a fully specified, competitive equilibrium model.

This article is motivated by the desire to apply the recursive, competitive equilibrium approach to the APT. In fact, the results also give predictions that make the model distinct from the static APT. In the static APT a oneperiod riskless asset serves as the zero-beta asset. In our model the zero-

${ }_{13}$ See Shanken (1985) for a discussion of the distinction among APT, equilibrium APT, and multi-beta CAPMs. 
beta asset is a long-lived asset rather than a one-period riskless asset. This parallels a result by Rubinstein (1981).

While the static APT, intertemporal APT, and ICAPM are, in principle, distinct, we argue that it may be hard in practice to distinguish them based on empirical tests. A weakness of all three of these models is that the ambiguity of the benchmark risk portfolios can lead to "overfitting" of the model to the data. The APT can be subject to overfitting because the pervasive factors are identified from asset return data that also are used to test the model. The ICAPM can be overfitted because one is free to use whatever state variables satisfy one's intuition as to "important" parameters of the representative investor's wealth function. The economist's intuition is likely to reflect, at least in part, his knowledge of the asset return data.

\section{Appendix}

\section{Proof of Theorem 1}

Our method of deriving asset prices uses standard results for the representative investor's intertemporal maximization problem [for example, see Harris (1987, pp. 95-97) or Sargent (1987, Chap. 3)]. We use the fact that, given our exchange economy and positive marginal utility of consumption, optimal consumption of the representative investor is equal to the aggregate dividend payment. We must show that the investor will choose to hold the market portfolio given the assumed price sequence. This will be true if and only if prices at time $t$ satisfy the following first-order condition:

$$
\mathbf{p}_{t} u^{\prime}\left(\mathbf{q}^{\prime} \mathbf{x}_{t}\right)=E_{t}\left[\sum_{s=1}^{\infty} \rho^{s} u^{\prime}\left(\mathbf{q}^{\prime} \tilde{\mathbf{x}}_{t+s}\right) \tilde{\mathbf{x}}_{t+s}\right]
$$

Dividing by $u^{\prime}\left(\mathbf{q}^{\prime} \tilde{\mathbf{x}}_{t}\right)$ and using the definition of $\tilde{\mathbf{x}}_{t+s}$ from Equation (1) gives

$$
\begin{aligned}
\mathbf{p}_{t}= & \mathbf{c}\left\{\frac{E_{t}\left[\sum_{s=1}^{\infty} \rho^{s} u^{\prime}\left(\mathbf{q}^{\prime} \tilde{\mathbf{x}}_{t+s}\right) \tilde{f}_{0 t+s-1}\right]}{u^{\prime}\left(\mathbf{q}^{\prime} \mathbf{x}_{t}\right)}\right\}+B\left\{\frac{E_{t}\left[\sum_{s=1}^{\infty} \rho^{s} u^{\prime}\left(\mathbf{q}^{\prime} \tilde{\mathbf{x}}_{t+s}\right) \tilde{\mathbf{f}}_{t+s}\right]}{u^{\prime}\left(\mathbf{q}^{\prime} \mathbf{x}_{t}\right)}\right\} \\
& +\frac{E_{t}\left[\sum_{s=1}^{\infty} \rho^{s} u^{\prime}\left(\mathbf{q}^{\prime} \tilde{\mathbf{x}}_{t+s}\right) \tilde{\boldsymbol{\epsilon}}_{t+s}\right]}{u^{\prime}\left(\mathbf{q}^{\prime} \mathbf{x}_{t}\right)}
\end{aligned}
$$

Note that $E_{t}\left[\tilde{\epsilon}_{t+1} \mid \mathbf{f}_{t+1}\right]=\mathbf{0}$ implies $E_{t}\left[\tilde{\boldsymbol{\epsilon}}_{t+s} \mid \mathbf{f}_{t+s}\right]=\mathbf{0}$, which, along with Assumption 6 , implies that the third additive term on the right-hand side of (A1) is identically zero. Using the definitions of $\pi_{0 t}$ and $\pi_{t}$ to restate (A1) gives the result. 


\section{Proof of Corollary 1}

By definition of return we have $r_{i t}=\left(p_{i t}+x_{i t}\right) / p_{i t-1}$. Using the formula for competitive equilibrium prices from Theorem 1 and the definition of $x_{i t}$ from Equation (1) gives

$$
r_{i t}=\frac{c_{i}\left(\pi_{0 t}+f_{0 t-1}\right)+\mathbf{B}_{i} \cdot\left(\boldsymbol{\pi}_{t}+\mathbf{f}_{t}\right)+\epsilon_{i t}}{c_{i} \boldsymbol{\pi}_{0 t-1}+\mathbf{B}_{i} \cdot \boldsymbol{\pi}_{t-1}}
$$

Note that, from Equation (2), $p_{i t}=c_{t} \boldsymbol{\pi}_{0 t}+\mathbf{B}_{i} \boldsymbol{\pi}_{t}$. Separating the additive terms in the expression above we obtain

$$
\begin{aligned}
r_{i t}= & \left(\frac{c_{i}}{p_{i t-1}}\right)\left(\pi_{0 t}+f_{0 t-1}\right)+\left(\frac{B_{i 1}}{p_{i t-1}}\right)\left(\pi_{1 t}+f_{1 t}\right) \\
& +\cdots+\left(\frac{B_{i k}}{p_{i t-1}}\right)\left(\pi_{k t}+f_{k t}\right)+\epsilon_{i t}^{*}
\end{aligned}
$$

Multiplying and dividing the first $k+1$ terms on the right-hand side of (A2) by $\pi_{0 t-1}, \pi_{1 t-1}, \ldots, \pi_{k t-1}$, respectively, gives

$$
\begin{aligned}
r_{i t}= & \frac{c_{i} \pi_{0 t-1}}{p_{i t-1}}\left(\frac{\pi_{0 t}+f_{0 t-1}}{\pi_{0 t-1}}\right)+\frac{B_{i 1} \pi_{1 t-1}}{p_{i t-1}}\left(\frac{\pi_{1 t}+f_{1 t}}{\pi_{1 t-1}}\right) \\
& +\cdots+\frac{B_{i k} \pi_{k t-1}}{p_{i t-1}}\left(\frac{\pi_{k t}+f_{k t}}{\pi_{k t-1}}\right)+\epsilon_{i t}^{*}
\end{aligned}
$$

Note that all these steps apply to portfolios as well as to assets. Using the definitions of $r_{0 t}, r_{m 1 t}, \ldots, r_{m k t}$ and finding their returns from (A3) gives

$$
r_{0 t}=\frac{\pi_{0 t}+f_{0 t-1}}{\pi_{0 t-1}}, r_{m 1 t}=\frac{\pi_{1 t}+f_{1 t}}{\pi_{1 t-1}} \ldots r_{m k t}=\frac{\pi_{k t}+f_{k t}}{\pi_{k t-1}}
$$

Inserting these portfolio returns into the right-hand side of (A3) and applying the definition of $\beta_{i j t}$ gives

$$
r_{i t}=\left(\frac{c_{i} \pi_{0 t-1}}{p_{i t-1}}\right) r_{0 t}+\beta_{i t t-1} r_{m 1 t}+\cdots+\beta_{i k t-1} r_{m k t}+\epsilon_{i t}^{*}
$$

Note that from the definition of $p_{i t}$ and $\beta_{i j t}$ we have $c_{i} \pi_{0 t} / p_{i t}=1-\beta_{i 1 t}-$ $\ldots-\beta_{i k r}$ Inserting this in (A4) produces

$$
\tilde{r}_{i t}=\tilde{r}_{0 t}+\beta_{i 1 t-1}\left(\tilde{r}_{m 1 t}-\tilde{r}_{0 t}\right)+\ldots+\beta_{i k t-1}\left(\tilde{r}_{m k t}-\tilde{r}_{0 t}\right)+\tilde{\epsilon}_{i t}^{*}
$$

Note that the assumptions underlying the factor structure of Equation (1) and Assumption 3 guarantee that $E\left(\tilde{\epsilon}_{i t}^{*} \mid r_{m 1 t}, \ldots, r_{m k t}\right)=0$. The assumption that $f_{0 t+s}$ and $\epsilon_{t}$ are independent for $s \geq 0$ also ensures that $E\left(\tilde{\epsilon}_{i t}^{*} \mid r_{0 t}, r_{m 1 t}\right.$, $\left.\ldots, r_{m k t}\right)=0$.

\section{Proof of Corollary 2}

Applying Theorem 1 and Corollary 1 we have

$$
\tilde{r}_{i t}-\tilde{r}_{0 t}=\beta_{i 1 t-1}\left(\tilde{r}_{m 1 t}-\tilde{r}_{0 t}\right)+\ldots+\beta_{i k t-1}\left(\tilde{r}_{m k t}-\tilde{r}_{0 t}\right)+\tilde{\epsilon}_{i t}^{*}
$$


where all terms are defined in Corollary 1 . Note that if $\pi_{j t} /\left(c_{i} \pi_{0 t}+\mathbf{B}_{i} \boldsymbol{\pi}_{t}\right)$ is constant through time, then so is $\beta_{i j}$. From the definition of $\pi_{0 t} \pi_{j t}$ in Theorem 1, and Assumption 8, we have

$$
\begin{aligned}
& \pi_{0 t}= \frac{E_{t}\left[\sum_{s=1}^{\infty} \rho^{s} e^{-\gamma \mathbf{q}^{\prime} \hat{\mathbf{x}}_{t+s}}\right]}{\left.e^{-\gamma \mathbf{q}^{\prime} \mathbf{x}_{t}}\right]} \\
& \pi_{t}=\frac{E_{t}\left[\sum_{s=1}^{\infty} \rho^{s} e^{-\gamma \mathbf{q}^{\prime} \tilde{\mathbf{x}}_{t+s}+\tilde{\mathbf{f}}_{t+s}}\right]}{e^{-\gamma \mathbf{q}^{\prime} \mathbf{x}_{t}}}
\end{aligned}
$$

Applying the assumption that $f_{0 t-1}$ and $\mathbf{f}_{t}$ have time-independent distributions,

$$
\pi_{0 t}=\frac{\rho e^{\gamma \mathbf{q}^{\prime} \mathbf{x} t} E\left[e^{-\gamma \mathbf{q}^{\prime} \tilde{\mathbf{x}}_{t+1}}\right]}{1-\rho} \quad \pi_{t}=\frac{\rho e^{\gamma \mathbf{q}^{\prime} \mathbf{x}_{t}} E\left[e^{-\gamma \mathbf{q}^{\prime} \mathbf{x}_{t+1}} \tilde{\mathbf{f}}_{t+1}\right]}{1-\rho}
$$

Note that the ratio $\pi_{j t} /\left(c_{i} \pi_{0 t}+\mathbf{B}_{i} \cdot \boldsymbol{\pi}_{t}\right)$ is time-invariant for all $i, j$.

\section{Proof of Corollary 3}

Using Assumption 10, we can write the dividend process as

$$
\mathbf{x}_{t}=\mathbf{c} f_{0 t-1}^{*}+B \mathbf{f}_{t}^{*}+\epsilon_{t}^{* *}
$$

where $f_{0 t-1}^{*}=\left(\mathbf{q}^{\prime} \mathbf{x}_{t-1}\right), \mathbf{f}_{t}^{*}=\mathbf{f}_{t}\left(\mathbf{q}^{\prime} \mathbf{x}_{t-1}\right)$, and $\boldsymbol{\epsilon}_{t}^{* *}=\boldsymbol{\epsilon}_{t}\left(\mathbf{q}^{\prime} \mathbf{x}_{t-1}\right)$. Note that using (A5) as a version of Equation (1), the dividend process $\mathbf{x}_{t}$ satisfies Assumptions 1-7. We can, therefore, infer from Theorem 1 that there exists a unique sequence of competitive equilibrium prices given by $\mathbf{p}_{t}=\mathbf{c} \pi_{0 t}+B \boldsymbol{\pi}_{t}$ where

$$
\pi_{0 t}=\frac{E_{t}\left[\sum_{s=1}^{\infty} \rho^{s} u^{\prime}\left(\mathbf{q}^{\prime} \tilde{\mathbf{x}}_{t+s}\right) \tilde{f}_{0 t+s-1}^{*}\right]}{u^{\prime}\left(\mathbf{q}^{\prime} \mathbf{x}_{t}\right)}
$$

and

$$
\pi_{t}=\frac{E_{t}\left[\sum_{s=1}^{\infty} \rho^{s} u^{\prime}\left(\mathbf{q}^{\prime} \tilde{\mathbf{x}}_{t+s}\right) \tilde{\mathbf{f}}_{t+s}^{*}\right]}{u^{\prime}\left(\mathbf{q}^{\prime} \mathbf{x}_{t}\right)}
$$

Using the assumed form of the utility function and the definitions of $f_{0 t-1}^{*}$ and $\mathbf{f}_{t}^{*}$, these become

$$
\pi_{0 t}=\frac{E_{t}\left[\sum_{s=1}^{\infty} \rho^{s}\left(\mathbf{q}^{\prime} \tilde{\mathbf{x}}_{t+s}\right)^{-\gamma}\left(\mathbf{q}^{\prime} \tilde{\mathbf{x}}_{t+s-1}\right)\right]}{\left(\mathbf{q}^{\prime} \mathbf{x}_{t}\right)^{-\gamma}}
$$




$$
\pi_{t}=\frac{E_{t}\left[\sum_{s=1}^{\infty} \rho^{s}\left(\mathbf{q}^{\prime} \tilde{\mathbf{x}}_{t+s}\right)^{-\gamma} \tilde{\mathbf{f}}_{t+s}\left(\mathbf{q}^{\prime} \tilde{\mathbf{x}}_{t+s-1}\right)\right]}{\left(\mathbf{q}^{\prime} \mathbf{x}_{t}\right)^{-\gamma}}
$$

For any $s \geq 1$ we can write $\mathbf{q}^{\prime} \mathbf{x}_{t+s}=\mathbf{q}^{\prime} \mathbf{x}_{t} \prod_{z=1}^{s}\left(1+\mathbf{q}^{\prime} B \mathbf{f}_{t+z}\right)$. Applying this to the expressions for $\pi_{0 t}$ and $\pi_{t}$ above,

$$
\begin{aligned}
& \pi_{0 t}=\frac{E_{t}\left[\sum_{s=1}^{\infty} \rho^{s}\left(\mathbf{q}^{\prime} \mathbf{x}_{t}\right)^{1-\gamma}\left[\prod_{z=1}^{s-1}\left(1+\mathbf{q}^{\prime} B \tilde{\mathbf{f}}_{t+z}\right)\right]^{1-\gamma}\left(1+\mathbf{q}^{\prime} B \tilde{\mathbf{f}}_{t+s}\right)^{-\gamma}\right]}{\left(\mathbf{q}^{\prime} \mathbf{x}_{t}\right)^{-\gamma}} \\
& \boldsymbol{\pi}_{t}=\frac{E_{t}\left[\sum_{s=1}^{\infty} \rho^{s}\left(\mathbf{q}^{\prime} \mathbf{x}_{t}\right)^{1-\gamma}\left[\prod_{z=1}^{s-1}\left(1+\mathbf{q}^{\prime} B \tilde{\mathbf{f}}_{t+z}\right)\right]^{1-\gamma}\left(1+\mathbf{q}^{\prime} B \tilde{\mathbf{f}}_{t+s}\right)^{-\gamma} \tilde{\mathbf{f}}_{t+s}\right]}{\left(\mathbf{q}^{\prime} \mathbf{x}_{t}\right)^{-\gamma}}
\end{aligned}
$$

Simplifying these expressions by collecting the $\left(\mathbf{q}^{\prime} \mathbf{x}_{t}\right)$ terms and using the fact that $\mathbf{f}_{t}$ is i.i.d. through time,

$$
\begin{aligned}
\pi_{0 t} & =\left(\mathbf{q}^{\prime} \mathbf{x}_{t}\right) \sum_{s=1}^{\infty} \rho^{s}\left\{E\left[\left(1+\mathbf{q}^{\prime} B \tilde{\mathbf{f}}\right)^{1-\gamma}\right]\right\}^{s-1} E\left[\left(1+\mathbf{q}^{\prime} B \tilde{\mathbf{f}}\right)^{-\gamma}\right] \\
\pi_{t} & =\left(\mathbf{q}^{\prime} \mathbf{x}_{t}\right) \sum_{s=1}^{\infty} \rho^{s}\left\{E\left[\left(1+\mathbf{q}^{\prime} B \tilde{\mathbf{f}}\right)^{1-\gamma}\right]\right\}^{s-1} E\left[\left(1+\mathbf{q}^{\prime} B \tilde{\mathbf{f}}\right)^{-\gamma \tilde{\mathbf{f}}}\right]
\end{aligned}
$$

Applying the rules for geometric series to these expressions,

$$
\begin{aligned}
\pi_{0 t} & =\frac{\rho \mathbf{q}^{\prime} \mathbf{x}_{t} E\left[\left(1+\mathbf{q}^{\prime} B \tilde{\mathbf{f}}\right)^{-\gamma}\right]}{1-\rho E\left[\left(1+\mathbf{q}^{\prime} B \tilde{\mathbf{f}}\right)^{1-\gamma}\right]} \\
\boldsymbol{\pi}_{t} & =\frac{\rho \mathbf{q}^{\prime} \mathbf{x}_{t} E\left[\left(1+\mathbf{q}^{\prime} B \tilde{\mathbf{f}}\right)^{-\gamma} \tilde{\mathbf{f}}\right]}{1-\rho E\left[\left(1+\mathbf{q}^{\prime} B \tilde{\mathbf{f}}\right)^{1-\gamma}\right]}
\end{aligned}
$$

Note that $\pi_{0 t}$ and $\pi_{t}$ have the same form as they do in Corollary 2 -a scalar function of $\mathbf{q}^{\prime} \mathbf{x}_{t}$ common to all the $\pi$ 's, multiplied by a time-invariant expression. By the same steps as in Corollary 2 it follows that we can write returns as

$$
r_{i t}-r_{0 t}=\beta_{i j}\left(r_{m 1 t}-r_{0 t}\right)+\cdots+\beta_{i k}\left(r_{m k t}-r_{0 t}\right)+\epsilon_{i t+1}^{*}
$$

where the $\beta_{i j}$ terms are time-invariant. Note also that

$$
\epsilon_{i t}^{*}=\frac{\epsilon_{i t}^{* *}}{c_{i} \pi_{0 t-1}+\mathbf{B}_{i} \cdot \pi_{t-1}}=\frac{\left(\mathbf{q}^{\prime} \mathbf{x}_{t-1}\right) \epsilon_{i t}}{\left(\mathbf{q}^{\prime} \mathbf{x}_{t-1}\right) \delta_{i}}=\frac{\epsilon_{i t}}{\delta_{i}}
$$

where $\delta_{i}$ is a time-invariant scalar. Hence $\epsilon_{i t}$ is conditionally homoskedastic by Assumption 12 . 


\section{References}

Bollerslev, T. P., R. F. Engle, and J. M. Wooldridge, 1988, "A Capital Asset Pricing Model with Time Varying Covariances," Journal of Political Economy, 96, 116-131.

Bossaerts, P., and R. C. Green, 1987, "Portfolio Separation, Aggregation and Equilibrium Rates of Return in a Recursive Economy," working paper, GSIA, Carnegie-Mellon University, March.

Bossaerts, P., and R. C. Green, 1988, "A General Equilibrium Model of Changing Risk Premia: Theory and Tests," working paper, GSIA, Carnegie-Mellon University, April.

Chamberlain, G., and M. Rothschild, 1983, "Arbitrage, Factor Structure, and Mean-Variance Analysis on Large Asset Markets," Econometrica, 51, 1281-1304.

Chen, N., 1983, "Some Empirical Tests of the Theory of Arbitrage Pricing," Journal of Finance, 38, 13931414 .

Connor, G., 1984, "A Unified Beta Pricing Theory," Journal of Economic Theory, 34, 13-31.

Connor, G., and R. A. Korajczyk, 1988, "Risk and Return in an Equilibrium APT: Application of a New Test Methodology," Journal of Financial Economics, 21, 255-289.

Constantinides, G. M., 1989, "Theory of Valuation: Overview and Recent Developments," in S. Bhattacharya and G. M. Constantinides (eds.), Theory of Valuation: Frontiers of Modern Financial Tbeory, Rowman \& Littlefield, Totowa, N.J.

Fama, E. F, and K. R. French, 1988, "Dividend Yields and Expected Stock Returns," Journal of Financial Economics, 22, 3-25.

Fama, E. F., and G. W. Schwert, 1977, "Asset Returns and Inflation," Journal of Financial Economics, 5 , $115-146$.

French, K. R., G. W. Schwert, and R. F. Stambaugh, 1987, "Expected Stock Returns and Volatility," Journal of Financial Economics, 19, 3-29.

Harris, M., 1987, Dynamic Economic Analysis, Oxford University Press, Oxford.

Huberman, G., and S. Kandel, 1987, "Mean-Variance Spanning," Journal of Finance, 42, 873-888.

Ingersoll, J. E., Jr., 1984, "Some Results in the Theory of Arbitrage Pricing," Journal of Finance, 39, 10211039 .

Jagannathan, R., and S. Viswanathan, 1988, "Linear Factor Pricing, Term Structure of Interest Rates and the Small Firm Anomaly," Working Paper 57, Department of Finance, Northwestern University, September.

Keim, D. B., and R. F. Stambaugh, 1986, "Predicting Returns in the Stock and Bond Markets," Journal of Financial Economics, 17, 357-390.

Kleidon, A. W., 1986, "Variance Bounds Tests and Stock Price Valuation Models," Journal of Political Economy, 94, 953-1001.

Lehmann, B. N., and D. M. Modest, 1988, "The Empirical Foundations of the Arbitrage Pricing Theory," Journal of Financial Economics, 21, 213-254.

Lo, A. W., and A. C. MacKinlay, 1988, "Stock Market Prices Do Not Follow Random Walks: Evidence from a Simple Specification Test," Review of Financial Studies, 1, 41-66.

Lucas, R. E., Jr., 1978, "Asset Prices in an Exchange Economy," Econometrica, 46, 1429-1445.

Merton, R. C., 1973, “An Intertemporal Capital Asset Pricing Model," Econometrica, 41, 867-887.

Ohlson, J. A., and M. B. Garman, 1980, "A Dynamic Equilibrium for the Ross Arbitrage Model," Journal of Finance, 35, 675-684.

Prescott, E. C., and R. Mehra, 1980, "Recursive Competitive Equilibrium: The Case of Homogeneous Households," Econometrica, 48, 1365-1379

Roll, R., and S. A. Ross, 1980, "An Empirical Investigation of the Arbitrage Pricing Theory," Journal of Finance, 35, 1073-1103. 
Ross, S. A., 1976, "The Arbitrage Theory of Capital Asset Pricing," Journal of Economic Theory, 13, 341360 .

Rothschild, M., and J. E. Stiglitz, 1970, "Increasing Risk: I. A Definition," Journal of Economic Theory, 2, $225-243$.

Rubinstein, M. E., 1981, "A Discrete-Time Synthesis of Financial Theory," in H. Levy (ed.), Researcb in Finance (vol. 3), JAI Press, Greenwich, Conn.

Sargent, T. J., 1987, Dynamic Macroeconomic Tbeory, Harvard University Press, Cambridge, Mass.

Shanken, J., 1985, "Multi-Beta CAPM or Equilibrium APT?: A Reply," Journal of Finance, 40, 1189-1196. 